\title{
Theta activity paradoxically boosts gamma and ripple frequency sensitivity in prefrontal interneurons Ricardo Martins Merino ${ }^{1,2,3,4,5}$, Carolina Leon-Pinzon ${ }^{1,2,3,5}$, Walter Stühmer ${ }^{3,5}$, Martin Möck ${ }^{6}$, Jochen F. Staiger ${ }^{6}$, Fred Wolf ${ }^{1,2,3,5,7,8}$, Andreas Neef $\mathbf{f}^{1,2,3,5,7,8^{*}}$
}

\author{
${ }^{1}$ Max Planck Institute for Dynamics and Self-Organization, Göttingen, Germany. \\ ${ }^{2}$ Göttingen Campus Institute for Dynamics of Biological Networks, Göttingen, Germany. \\ ${ }^{3}$ Bernstein Center for Computational Neuroscience Göttingen, Germany. \\ ${ }^{4}$ Göttingen Graduate Center for Neurosciences, Biophysics, and Molecular Biosciences, University of \\ Göttingen, Germany. \\ ${ }^{5}$ Max Planck Institute of Experimental Medicine, Göttingen, Germany. \\ ${ }^{6}$ Institute for Neuroanatomy, University Medical Center Göttingen, Georg-August-University, \\ Göttingen, Germany. \\ ${ }^{7}$ Institute for Nonlinear Dynamics, Georg-August University School of Science, Göttingen, Germany. \\ ${ }^{8}$ Center for Biostructural Imaging of Neurodegeneration, Göttingen, Germany. \\ *e-mail: aneef@gwdg.de
}

\begin{abstract}
Fast oscillations in cortical circuits critically depend on GABAergic interneurons. Which interneuron types and populations can drive different cortical rhythms, however, remains unresolved and may depend on brain state. Here, we measured the sensitivity of different GABAergic interneurons in prefrontal cortex under conditions mimicking distinct brain states. While fast-spiking neurons always exhibited a wide bandwidth of around $400 \mathrm{~Hz}$, the response properties of spike-frequency adapting interneurons switched with the background input's statistics. Slowly fluctuating background activity, as typical for sleep or quiet wakefulness, dramatically boosted the neurons' sensitivity to gamma- and ripple-frequencies. A novel timeresolved dynamic gain analysis revealed rapid sensitivity modulations that enable neurons to periodically boost gamma oscillations and ripples during specific phases of ongoing lowfrequency oscillations. This mechanism presumably contributes substantially to crossfrequency coupling and predicts these prefrontal interneurons to be exquisitely sensitive to high-frequency ripples, especially during brain states characterized by slow rhythms.
\end{abstract}

\section{MAIN TEXT}

\section{Introduction}

Collective rhythmic activity is implicated in brain functions from sensory information processing to memory consolidation, often with higher frequency activity bouts locked onto lower frequencies (1-3). While the mechanism behind this cross-frequency-coupling is unclear (3), the initiation and maintenance of gamma band $(30-150 \mathrm{~Hz})$ oscillations are closely associated with fast-spiking (FS), parvalbumin-positive interneurons $(4,5)$. When driven with frequency chirps, and as a result of intrinsic membrane properties, FS neurons fire more robustly at higher input frequencies than spike-frequency adapting (AD), somatostatinpositive interneurons, which are most responsive to lower frequencies (6). Nevertheless, recent studies strongly suggest that, under certain conditions, somatostatin-positive interneurons are crucial for gamma oscillations (7-9). Could the spectral sensitivity of different interneuron populations perhaps be itself state-dependent? Here we characterized 
cortical GABAergic interneurons at different in vivo-like working points by measuring their dynamic gain (10-14).

Dynamic gain quantifies how input in different frequency bands modulates population firing under in vivo-like conditions of fluctuating background input. To probe the potential impact of different brain states on spectral sensitivity, we used different types of background inputs that mimic the strength and timescales of correlations in background input across brain states (15). We find that both FS and AD interneuron populations can have remarkably wide bandwidths (up to about $500 \mathrm{~Hz}$ ), making them capable of tracking fast input frequencies well into the range of sharp wave-ripples.

Moreover, our results uncover unanticipated flexibility in AD neurons, which can massively shift their frequency preference, specifically engaging or disengaging with high-frequency rhythms, such as gamma and sharp wave-ripples. The presence or absence of slowlycorrelated input drives this sensitivity shift, which can occur within $50 \mathrm{~ms}$, in phase with an ongoing slow rhythm. This observation offers a mechanistic explanation for theta-gamma cross-frequency coupling.

\section{Results}

$\mathrm{AD}$ and FS (Figs. 1A and 1B) are the most common firing patterns of somatostatin- and parvalbumin-positive interneurons, respectively (16). Their spectral selectivity has been investigated through sub- and supra-threshold cellular responses to simple, purely sinusoidal inputs (6) (Figs. 1C and 1D). However, in vivo, even when activity on the population level is periodic, the firing of individual neurons appears stochastic, driven by noisy, fluctuating inputs rather than pure sinusoids $(13,17)$. We thus probed the spectral sensitivity of mouse layer $2 / 3$ prefrontal FS and AD interneuron populations under naturalistic operating conditions (Fig. 1E). These dynamic gain measurements require precise control over the neurons' input to a degree that cannot be attained in vivo. We, therefore, used patch-clamp recordings in current-clamp mode in acute prefrontal slices to establish two different regimes of fluctuating input, distinguished by the correlation time $\tau$ : the first case, $\tau=5 \mathrm{~ms}$, mimics the case of completely asynchronous population activity, when the decay time-constant of synaptic currents is the only source of input correlations (Fig. 1E, black traces). The other input, characterized by a much slower $25 \mathrm{~ms}$ correlation time, mimics brain states with population activity exhibiting slow fluctuations, such as quiet wakefulness or slow-wave sleep (15) (Fig. 1E, gray traces).
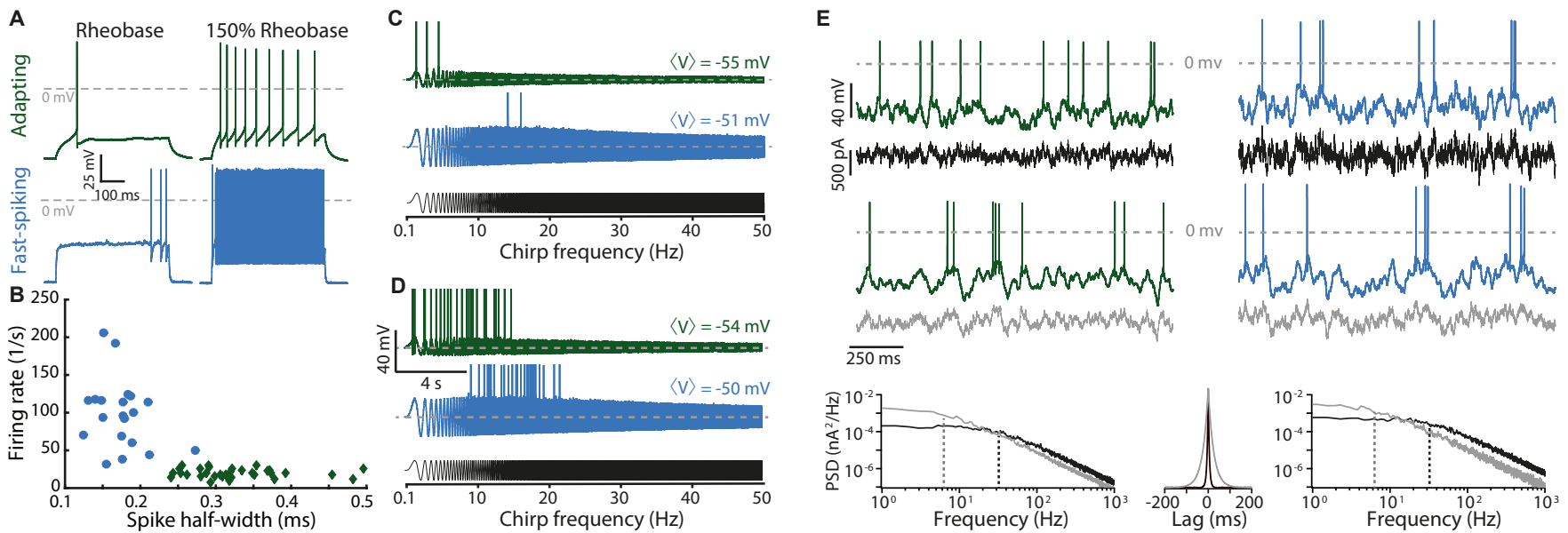

Fig 1. Characterization of neocortical adapting and fast-spiking interneurons. (A) Square pulses of $500 \mathrm{~ms}$ were used to determine the recorded neuron's firing pattern at the $150 \%$ rheobase level. Shown are representative responses of spike-frequency adapting ( $\mathrm{AD}$, green) and fast-spiking (FS, blue) neurons at rheobase and $150 \%$ rheobase. (B) Spike half-width and firing rate allow a clear distinction between these cell types. (C) Frequency chirp currents (black) have been used to characterize the spectral sensitivity of neurons. They yield action potentials (shown clipped) at lower input frequencies for AD neurons than for FS neurons. (D) A slight increase in the offset current, resulting in only a $1 \mathrm{mV}$ depolarization, results in overlapping bandwidths for AD and FS neurons, indicating substantial uncertainty in chirp-based characterizations. (E) We assessed neuronal encoding performance in two in vivo-like regimes, distinguished by the correlation time of the fluctuating stimuli $(\tau=5 \mathrm{~ms}$, 
black, and $\tau=25 \mathrm{~ms}$, gray). The stimulus amplitude at each trial was adjusted to achieve a target operating point (characterized by firing rate and spike train irregularity; see Methods). The corresponding voltage traces of AD and FS neurons are shown above the stimuli, and the power spectral densities (PSDs) and autocorrelations of the inputs are shown at the bottom. The dashed lines in the PSDs indicate the cut-off frequencies $(32 \mathrm{~Hz}$ and $6.4 \mathrm{~Hz})$ corresponding to the correlation times of the different inputs.

\section{Input correlations determine frequency selectivity}

The spectral sensitivity of interneurons was markedly different from their chirp responses, and for $\mathrm{AD}$ cells, it changed drastically between the two conditions (Figure $2 \mathrm{~A}$ ). In the asynchronous regime, $\mathrm{AD}$ neurons respond preferentially to slow components, with the highest sensitivity in the $2-4 \mathrm{~Hz}$ range (mean dynamic gain $=119 \mathrm{~Hz} / \mathrm{nA}, 95 \%$ bootstrap confidence interval: $[117$, 122]). The average gain in the gamma range (Fig. $2 \mathrm{~A}$, shaded region) reaches only $62 \%$ of the average at lower frequencies $(<20 \mathrm{~Hz})(64 \mathrm{~Hz} / \mathrm{nA}[63,65]$ vs $103 \mathrm{~Hz} / \mathrm{nA}[101,105])$. These values mean that the addition of a small, $10 \mathrm{pA}$ sinusoidal modulation (equivalent in magnitude to a single synaptic event) on top of the irregularly fluctuating background input would modulate the AD population's firing rate by $1.2 \mathrm{~Hz}$ in response to a superimposed $3 \mathrm{~Hz}$ input, but it would modulate the firing rate only by $0.6 \mathrm{~Hz}$ for $60 \mathrm{~Hz}$, indicating a clear preference for lower frequencies. This preference, however, changed completely when AD neurons were exposed to slowly fluctuating input such that their preferred frequency shifted from $3 \mathrm{~Hz}$ to $200 \mathrm{~Hz}$. The gain at 2-4 Hz dropped from $119 \mathrm{~Hz} / \mathrm{nA}[117,122]$ to $91 \mathrm{~Hz} / \mathrm{nA}[89,92]$, and the gain at 200 $\mathrm{Hz}$ increased from $74 \mathrm{~Hz} / \mathrm{nA}[72,76]$ to $119 \mathrm{~Hz} / \mathrm{nA}[113,124]$. Figure $2 \mathrm{~B}$ demonstrates the occurrence of this shift in two individual AD neurons. With this abrupt change in frequency preference, $\mathrm{AD}$ neurons in the synchronous regime become more sensitive to gamma input than to lower frequencies (average gains: $97 \mathrm{~Hz} / \mathrm{nA}[94,101]$ vs $81 \mathrm{~Hz} / \mathrm{nA}[80,83]$ ). Altogether, these data reveal that, during network states characterized by slow background fluctuations, AD cells tune themselves to gamma and higher-frequency input. FS interneurons, on the other hand, preferentially transmit high frequencies irrespective of the input correlations, with a maximum sensitivity around 200-250 Hz (Fig. 2C). Both, FS interneurons and, given sufficiently slow input components, $\mathrm{AD}$ interneurons have a remarkably wide bandwidth, with a high-frequency limit well above $400 \mathrm{~Hz}$, an order of magnitude higher than expected from their chirp-responses.
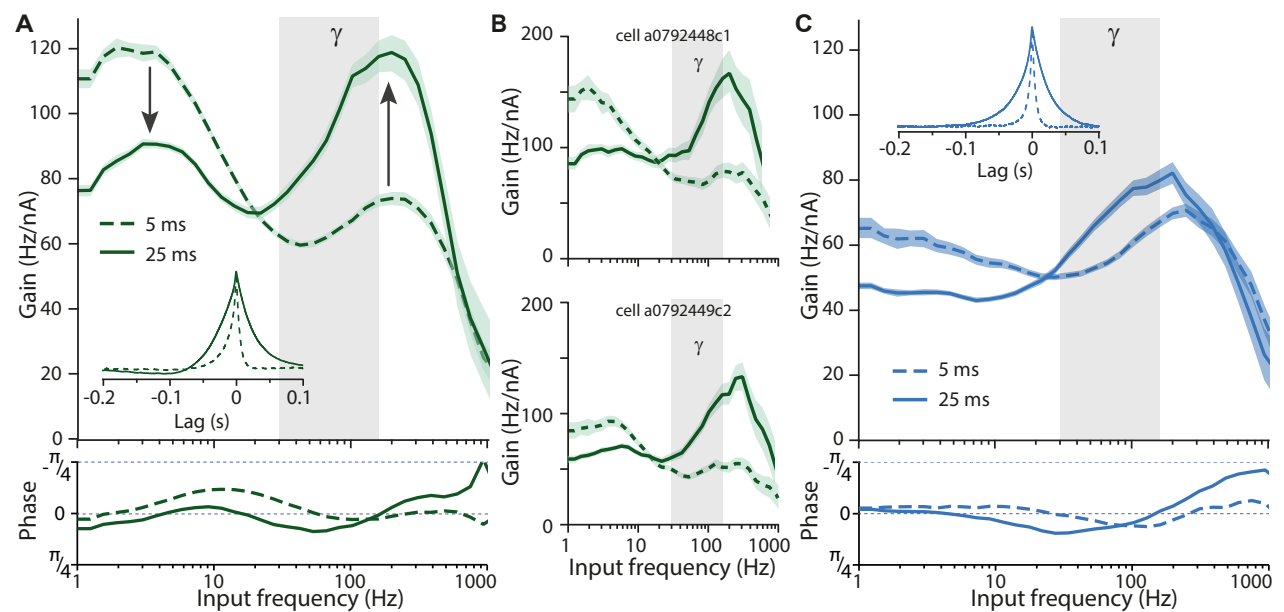

Fig. 2. Spectral selectivity of AD neurons drastically shifts for different background fluctuations. (A) (top) Gain of AD neurons tested with inputs with two different correlation times. Under fast background input, $\tau=5 \mathrm{~ms}$ (dashed lines, $\mathrm{n}=12$ ), $\mathrm{AD}$ neurons modulate their firing rate strongest in response to lower frequencies. Under slow background, $\tau=25 \mathrm{~ms}$ (continuous line, $\mathrm{n}=10$ ), the frequency preference shifts (arrows) and the firing rate is modulated mainly by high frequencies. Mean firing rate and coefficient of variation of the interspike intervals were $4.0 \mathrm{~Hz} \pm 0.2$ and $0.99 \pm 0.02$ (5 ms input) and $3.7 \mathrm{~Hz} \pm 0.2$ and $1.03 \pm 0.05$ (25 ms input), respectively. Inset: spike-triggered average input across all recorded cells tested with the same correlation times. Gains were calculated by taking the ratio of the Fourier transforms of the spike-triggered average and of the autocorrelation function of the input. Gray columns represent the gamma frequency band, and the shaded region around gain curves represents the $95 \%$ bootstrap confidence interval. (Bottom) Phase of firing rate modulation with respect to input. No substantial phase-delays are associated with action potential generation. (B) Individual gain curves of two AD cells from $\mathbf{A}$ for both correlation times. The drastic shift in frequency preference is clearly visible at the single-cell level. 

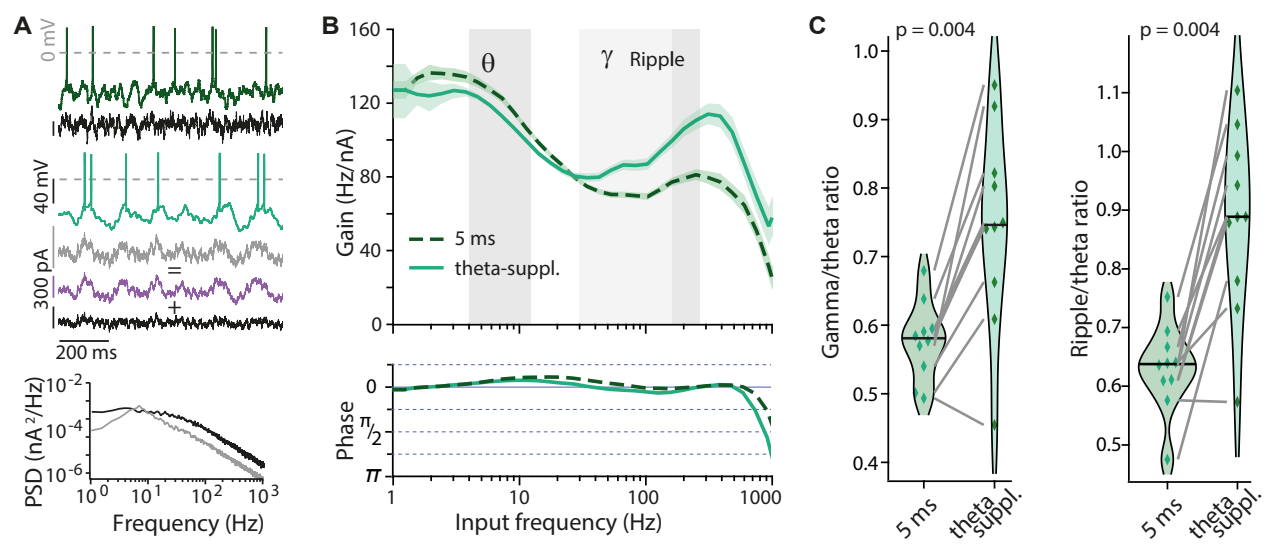

Figure 3. Increasing theta input to AD neurons boosts sensitivity to gamma and ripple frequencies. (A) Sample stimuli and voltage traces (dark green, $5 \mathrm{~ms}$ input; light green, theta-supplemented input) and power spectral density of noisy inputs with $\tau=5 \mathrm{~ms}$ (black) and theta-supplemented $5 \mathrm{~ms}$ input (gray). Thetasupplemented input was constructed by adding a theta bandpass filtered white noise input (purple) to the $5 \mathrm{~ms}$ input. Grand-averaged firing rate and coefficient of variation of the interspike intervals were $4.58 \mathrm{~Hz} \pm 0.23$ and $0.97 \pm 0.03$ ( $5 \mathrm{~ms}$ input) and $4.78 \mathrm{~Hz} \pm 0.26$ and $0.92 \pm 0.04$ (theta-supplemented $5 \mathrm{~ms}$ input). (B) (top) Gain of $\mathrm{AD}$ cells tested with both, $\tau=5 \mathrm{~ms}$ (dashed line) and theta-supplemented $5 \mathrm{~ms}$ (continuous line) inputs $(\mathrm{n}=10)$. Boosting theta in the input paradoxically reduces the sensitivity of $\mathrm{AD}$ neurons to this frequency band while promoting sensitivity to gamma and ripple frequencies $(150-250 \mathrm{~Hz})$. (Bottom) The phase of firing rate modulation with respect to the input. No substantial phase-delays are associated with action potential generation, even though the gain magnitude is modulated. $(\mathbf{C})$ Ratios between average gains at gamma and theta (left) and ripple and theta (right) for the individual neurons (diamonds). Both ratios increase when theta power in the input is increased. Gamma/theta ratio for $\tau=5 \mathrm{~ms}: 0.56 \pm 0.02$ vs theta-supplemented $5 \mathrm{~ms}: 0.74 \pm 0.05, \mathrm{n}=10$ (paired sample, twosided Wilcoxon signed-rank test, $\mathrm{W}=1, \mathrm{p}=0.004)$. Ripple/theta ratio for $\tau=5 \mathrm{~ms}: 0.63 \pm 0.02$ vs thetasupplemented $5 \mathrm{~ms}: 0.88 \pm 0.05, \mathrm{n}=10$ (two-sided Wilcoxon signed-rank test, $\mathrm{W}=0, \mathrm{p}=0.002$ ). Violin plots show the medians as bars. Numbers are given as mean \pm SEM

\section{Theta phase determines gamma sensitivity}

The dynamic gain curves above are based on the timing of all action potentials (APs) fired during a long stimulus period. They represent the average frequency selectivity for a given input statistics and allowed us to detect the boosting for gamma- and ripple frequencies during 30 second long periods of theta-dominated input. During in vivo activity, however, short gammabursts or ripples occur phase-locked to slower rhythms, consistent with the idea that neurons might be recruited to high-frequency rhythms within a few dozen milliseconds. Specifically, theta-gamma cross-frequency coupling suggests a modulation of gamma sensitivity throughout the phase of the ongoing theta rhythm. To test whether AD neurons indeed display such a 
180

modulation, we developed a time-resolved decomposition of the dynamic gain. To this end, we reanalyzing the data obtained with the theta-supplemented stimulus (Fig. 3), we determined the phase $\varphi_{\theta}$ of the stimulus' theta band at each AP time. We sorted the APs into three groups, according to $\varphi_{\theta}$. The boundaries between groups, $\varphi_{\theta}=-0.017$ and $\varphi_{\theta}=0.476$, were chosen so that each group contained one-third of all APs (Fig. 4A and B, Methods). For each group, the average gain across all ten neurons was determined as before (Fig. 4C). As expected for a meaningful decomposition, the three gain components combined to replicate the overall dynamic gain (Inset in fig. 4C). For each neuron, we calculated three dynamic gain curves, each derived from all the APs belonging to one $\varphi_{\theta}$ group. The neuron's ability to lock to gamma rhythms was quantified as the average gain value in the gamma range $(30-150 \mathrm{~Hz})$. Across the ten neurons, the median value of this gamma sensitivity increased from $27.4 \mathrm{~Hz} / \mathrm{nA}$ to 37.5 $\mathrm{Hz} / \mathrm{nA}$ as $\varphi_{\theta}$ goes from the first to the third group, revealing a theta-phase dependent locking of APs to gamma inputs (Fig. 4D). The substantially increased gamma sensitivity for APs fired later during a theta cycle indicates that $\mathrm{AD}$ neurons' frequency tuning changes within a quarter of a theta cycle, i.e., within less than $50 \mathrm{~ms}$.
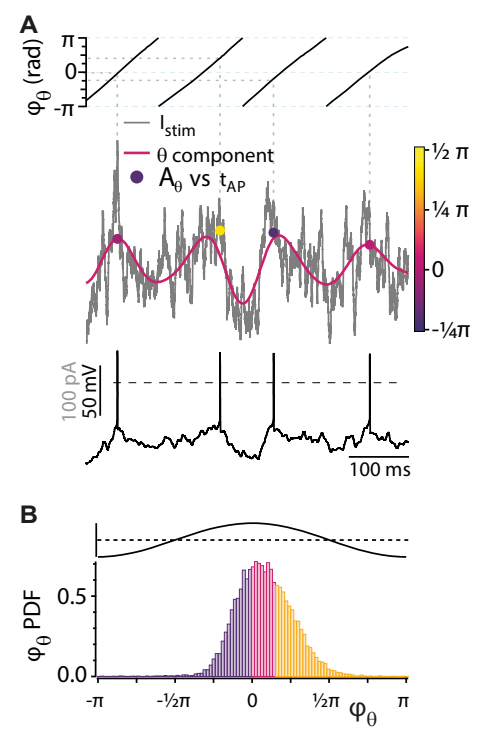
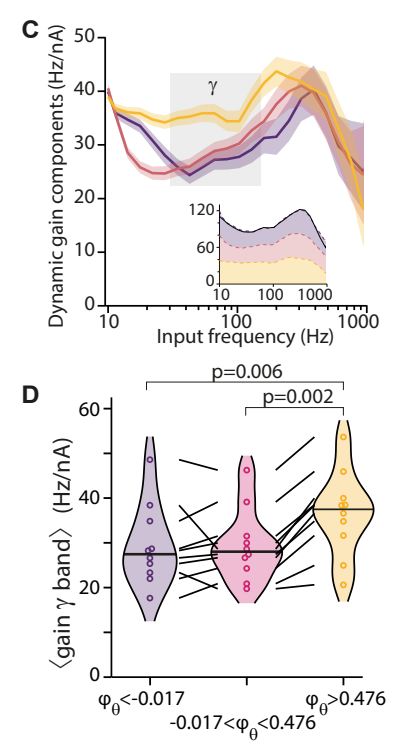

195 Fig. 4. Sensitivity to gamma frequencies is 196 modulated during progression through the 197 theta cycle. (A) Analysis of theta components 198 showing the membrane voltage (bottom, black), 199 input current (grey), its theta component 200 (magenta, see Methods), and points indicating 201 the time of action potentials, plotted against the 202 instantaneous theta amplitude $\mathrm{A}_{\theta}$ obtained by 203 Hilbert-Analysis. The color code corresponds to 204 the theta phase ( $\varphi_{\theta}$, top). (B) A probability 205 density plot of $\varphi_{\theta}$ with three differently colored 206 phase intervals. Each interval contains one-third 207 of all APs. The top trace indicates the cosine 208 relation between the theta component's phase 209 and amplitude. (C) The dynamic gain curves of 210 the three components (color code as in B) have distinctive frequency dependencies. Their 95\% confidence intervals (shaded) separate in the beta $(12-30 \mathrm{~Hz})$ and gamma frequency bands. In the inset, the three components are stacked. Their sum closely reproduces the overall dynamic gain obtained with the theta-supplemented input from fig. 3 B (re-ploted in black in the inset) (D) AD neurons' sensitivity to gamma frequencies (Methods) increases during the theta cycle from the lower two $\varphi_{\theta^{-}}$ intervals: $29.4 \pm 2.9 \mathrm{~Hz} / \mathrm{nA}$ (purple, left) and $29.4 \pm 2.6 \mathrm{~Hz} / \mathrm{nA}$ (magenta, center), to the APs with the highest $\varphi_{\theta}$ values: $36.5 \pm 3.0 \mathrm{~Hz} / \mathrm{nA}$ (orange, right). Gamma sensitivity in the latest interval (orange) is significantly higher than in the first (purple, $\mathrm{W}=2, \mathrm{p}=0.006$ ) and the second (magenta, $\mathrm{W}=0, \mathrm{p}=0.002$ ), based on paired sample, twosided Wilcoxon signed-rank tests.

\section{Discussion}

Our study reveals a novel type of dynamic regulation of AD neuron's response properties. When the neurons' input fluctuates rapidly, as during active wakefulness, our data support the traditional picture in which AD neurons preferentially encode low-frequency input, and FS neurons encode high-frequency $(>30 \mathrm{~Hz})$ input. A drastic change in the frequency preference of $\mathrm{AD}$ neurons occurs, however, when input correlations are slow, as during brain states featuring low-frequency dominated local field potentials. Under such conditions, AD neurons react preferentially to gamma and ripple frequencies. Our findings thus uncover an unanticipated flexibility of interneuron function that allows brain states to tune AD neuron population coding and might underlie their reported contribution to gamma oscillations $(7,9)$.

Previously, dynamic gain curves were studied as essentially static properties, determined from minute-long stimuli of stationary stochastic properties. Our time-resolved analysis revealed 
that $\mathrm{AD}$ neurons rapidly respond to fluctuations in input statistics, increasing their dynamic gain in the gamma-band by $50 \%$ within a few dozen milliseconds. Our decomposition approach provides a powerful extension to current population encoding analyses. It allows, for instance, a quantitative comparison between the encoding capacity of APs within ripples vs outside ripples, or of AP duplets as compared to isolated APs.

Increased encoding of high frequencies $(>30 \mathrm{~Hz})$ during particular phases of strong, slow (theta) components (Figs. 3, 4) closely matches the phenomenon of theta-gamma crossfrequency coupling (18). In the dynamics of recurrent local circuits, the dynamic gain is a main component to the feedback gain that determines whether a collective oscillation is amplified or dies out. In theoretical studies of population oscillations in synchronous (2) or asynchronous (19) network states, the magnitude and the phase of the dynamic gain are key determinants of oscillations strength and frequency (20). Therefore, the small phase-delays associated with AP generation (Figs. 2A, 2C and 3B) and the input-dependent increase in gain magnitude predict a boost of gamma oscillation amplitude in the presence of theta-frequency input components and in particular during late theta-phases. The dynamic tuning of spectral sensitivity in phase with slow input fluctuations is, to our knowledge, the first mechanism coupling gamma amplitude to theta oscillations that is based on cellular electrophysiological properties.

The wide bandwidth of AD and FS neurons of up to $500 \mathrm{~Hz}$ and a maximal sensitivity reached around $200 \mathrm{~Hz}$ is by itself a striking phenomenon. In cortical pyramidal neurons, high bandwidth dynamic gain is known to mediate the sub-millisecond precision of population coding for input changes (21), but what function could a narrow preference band at around $200 \mathrm{~Hz}$ serve? Retrieval and consolidation of episodic memory require a complex and precise replay of activity by cell assemblies in the form of high-frequency sharp wave-ripples (150$250 \mathrm{~Hz}$ ). Intriguingly, these occur specifically during periods of synchronous network activity, such as during slow-wave sleep or quiet wakefulness (22), when, as we showed, AD and FS neurons are most sensitive to high frequencies. Given the input-dependent selectivity switch in $\mathrm{AD}$ neurons, slow oscillations may, in general, boost high-frequency sensitivity of interneurons and specifically allow $\mathrm{AD}$ neurons to tune in to ripple-related inputs and disinhibit cortical circuits in a precisely timed manner.

\section{Materials and Methods}

\section{Animals and slice preparation}

All experiments were performed in accordance with institutional and state regulations (Niedersächsisches Landesamt für Verbraucherschutz und Lebensmittelsicherheit). Experiments were performed in 3 to 8-week-old mice of either sex from five different mouse lines. Two lines target mostly AD interneurons: GIN (FVB-Tg(GadGFP)45704Swn, The Jackson Lab \#003718) and SOMCrexAi9 (Ssttm2.1(cre)Zjh/J, The Jackson Lab \#013044, crossbred with B6.Cg-GT(ROSA)26Sor^tm9(CAG-tdTomato)Hze/J, The Jackson Lab \#007909); and three lines target mostly FS interneurons: PVCre (23), PVCrexAi32 (PVCre crossbred with B6;129S-Gt(ROSA)26Sortm32(CAG-COP4*H134R/EYFP)Hze/J, The Jackson Lab \# 012569), and Nkx2.1CreERxAi14 (Nkx2-1 tm1.1(cre/ERT2)Zjh/J, The Jackson Lab \# 014552, crossbred with B6;129S6-Gt(ROSA)26Sortm14(CAG-tdTomato)Hze/J, The Jackson Lab \# 007908). Animals were kept in standard 12h light regime with water and food ad libidum. Animals were intraperitoneally-injected with a mixture of ketamine and xylazine in PBS (respectively 100 and $20 \mathrm{mg} / \mathrm{kg}$ of body weight) and decapitated. The brain was quickly removed and kept in ice-cold, carbogen-saturated cutting solution containing, in $\mathrm{mM}$, $125 \mathrm{NaCl}, 2.5 \mathrm{KCl}, 26 \mathrm{NaHCO}_{3}, 1.25 \mathrm{NaH}_{2} \mathrm{PO}_{4}, 0.4$ Ascorbic Acid, 4 Na-Lactate, 25 Glucose, $1 \mathrm{MgCl}_{2}, 2 \mathrm{CaCl}_{2}(\sim 315 \mathrm{mOsm}, \mathrm{pH}$ 7.4). 300- $\mu \mathrm{m}$-thick coronal neocortical slices were made in a VT1200S Vibratome (Leica) and incubated at $35^{\circ} \mathrm{C}$ in carbogen-saturated 
recording solution (aCSF, in mM: $125 \mathrm{NaCl}, 4 \mathrm{KCl}, 26 \mathrm{NaHCO}_{3}, 10$ glucose, $1.3 \mathrm{MgCl}_{2}, 2$ $\mathrm{CaCl}_{2}$ ) until recorded.

\section{Patch-clamp recordings}

One slice at a time was transferred to a heated recording chamber (PH6 and RC-27L, Warner Instruments) and mechanically stabilized with a slice hold-down (SHD-27LH/15, Warner Instruments). Throughout the experiment, the slice was gravitationally perfused with warm aCSF through an in-line heater (HPT-2, Alasciences) at a 1-2.5 ml/min flow rate. Both the recording chamber and the in-line heater were controlled by a TC-20 temperature controller (NPI electronics). Temperature settings were adjusted so that a thermistor measured a target temperature of $36 \pm 1^{\circ} \mathrm{C}$ at the slice position. Slices were visualized in an Axio Examiner.D1 microscope (Zeiss) equipped with a W Plan-Apochromat 40x/1.0 DIC objective. Cells were visualized with infrared differential interference contrast optics (Zeiss), and fluorescent signal was imaged with a multi-wavelength LED source (pE-4000, CoolLed) and a CCD camera (MD061RU-SY, Ximea). 4-6 MOhm pipettes were prepared from borosilicate glass capillaries (PG10165-4, World Precision Instruments) in a vertical puller (PIP 6, HEKA). Internal solution contained, in $\mathrm{mM}, 135 \mathrm{~K}$-Gluconate, $10 \mathrm{KCl}, 4 \mathrm{NaCl}$, $0.1 \mathrm{Na}$ EGTA, $1 \mathrm{Mg}$-ATP, 0.3 Na-GTP, 10 Hepes, $0.5 \mathrm{Na}_{2}$-Phosphocreatine and 0.2\% (w/v) biocytin (285-290 MOsm, $\mathrm{pH}$ adjusted to 7.3). Whole-cell current-clamp recordings were made in an EPC-10 Double amplifier controlled by Patchmaster (Heka). Fast and slow capacitances and series resistance were carefully adjusted in voltage-clamp mode before recording; fast capacitances while in on-cell configuration, slow capacitances after achieving whole-cell configuration. Series resistance was $90-100 \%$ compensated with a feedback time constant of $10 \mu \mathrm{s}$. Voltage signals were low-pass filtered at $8.8 \mathrm{kHz}$ and digitized at $100 \mathrm{kHz}$. Data analyses were performed in custom-written Matlab 2014b (Mathworks) and Igor Pro 8 (Wavemetrics) programs. Liquid junction potential of $-14 \mathrm{mV}$ was not corrected. All experiments were performed in the presence of blockers of GABA receptors (picrotoxin, $30 \mu \mathrm{M}$, Sigma) and glutamate receptors (NBQX, $10 \mu \mathrm{M}$, Tocris; and DL-AP5, $30 \mu \mathrm{M}$, Sigma).

\section{Characterization of action potential firing patterns}

Layer $2 / 3$ interneurons were identified via fluorescence imaging. In order to identify their electrical type, 500-ms-long current steps were applied. Current amplitude was increased in 15 pA steps until at least 1.5 times rheobase, the level at which the characterization of the firing pattern was made. Only cells exhibiting clear fast-spiking (including stuttering cells) and adapting electrical types were included in the analysis.

\section{Dynamic gain calculation}

Population frequency-response characterization was restricted to layer $2 / 3$ prefrontal FS and $\mathrm{AD}$ interneurons and was assessed as previously described $(11,12)$. This analysis aims to achieve an in vivo-like operating point, mimicking a situation in which a high rate of synaptic inputs provides a continuously changing net background current, and a neurons' firing is driven not by the average input but by its transient depolarizing excursions (17). Fluctuating current inputs were synthesized as Ornstein-Uhlenbeck noises $x(t)$ with either 5 or $25 \mathrm{~ms}$ correlation time. These values were chosen to approximate the case of uncorrelated inputs filtered through the synaptic currents' decay time-constants $(5 \mathrm{~ms})$ or the case of slow temporal correlations in the input due to correlated network activity $(25 \mathrm{~ms})$. Inputs' standard deviation was adjusted to obtain similar firing rates (around $4 \mathrm{~Hz}$ ) and coefficients of variation of the interspike intervals (around 1) for both correlation times. Neurons were first depolarized to $-60 \mathrm{mV}$ with DC current and different realizations of the fluctuating noise were injected in 30 -s-long episodes, separated by 15 -s-long resting, for as long as the recording did not display signs of deterioration, such as baseline drifts or spike overshooting to positive voltages less than $20 \mathrm{mV}$. For experiments presented in figure 3, a theta-power enhanced stimulus was created by adding a $4-12 \mathrm{~Hz}$ bandpass filtered white noise to the $5 \mathrm{~ms}$ input. The standard deviation of the bandpass filtered signal was normalized to two times the standard deviation of the $5 \mathrm{~ms}$ signal. APs were detected as $0 \mathrm{mV}$ crossings on the voltage trace and the AP times 
were annotated. From these, a spike-triggered average input current (STA) was obtained by summing up 1-s-long stimulus segments centered on the AP times for all cells of a given condition and dividing by the total number of APs.

The complex dynamic gain function $G(f)$ was calculated as the ratio of the Fourier transform of the STA, $F|\mathrm{STA}|$, and the Fourier transform of the autocorrelation of the stimulus, $F\left|\mathrm{c}_{\mathrm{ss}}(\tau)\right|$ where

$$
c_{S S}(\tau)=\langle x(t) x(t+\tau)\rangle
$$

and $\tau$ denotes the time lag. In order to improve signal-to-noise ratio, $G(f)$ was filtered in the frequency domain by a Gaussian filter $w\left(f^{\prime}\right)$ centered at frequency $f^{\prime}=f$ and a frequencydependent window size with standard deviation of $f / 2 \pi$.

$$
w\left(f^{\prime}\right)=\frac{1}{\sqrt{2 \pi}\left(\frac{f}{2 \pi}\right)} \exp \left[\frac{-1}{2}\left(\frac{f^{\prime}-f}{f / 2 \pi}\right)^{2}\right]
$$

The filtered dynamic gain function $G_{w}(f)$ thus becomes

$$
G_{w}(f)=\frac{\int G\left(f^{\prime}\right) \cdot w\left(f^{\prime}\right) \cdot d f^{\prime}}{\int w\left(f^{\prime}\right) \cdot d f^{\prime}}
$$

The magnitude and phase of this filtered, complex dynamic gain function are reported in figures 2 and 3. For the dynamic gains reported in figure 2, the data comprise of: for $\mathrm{AD}$ neurons, 19563 spikes from 12 cells and 20427 spikes from 10 cells $(5 \mathrm{~ms}$ and $25 \mathrm{~ms}$ respectively), and, for FS neurons, 9792 spikes from 7 cells and 15023 spikes from 9 cells (5 $\mathrm{ms}$ and $25 \mathrm{~ms}$, respectively). Five of the $10 \mathrm{AD}$ neurons tested with $5 \mathrm{~ms}$-correlated stimuli were also tested with the theta-supplemented $5 \mathrm{~ms}$ input. In addition to these, another 5 were used to obtain the gains in figure 3 (14847 spikes, for $5 \mathrm{~ms}$ stimulus and 18067 spikes for theta-supplemented $5 \mathrm{~ms}$ stimulus). Confidence intervals were obtained by bootstrap resampling. 2000 bootstrapped gain curves were calculated from the same number of STAs obtained by randomly sampling from all STAs used in the population gain calculation. The confidence intervals are defined as the $2.5^{\text {th }}$ and $97.5^{\text {th }}$ percentiles at each frequency point in the 2000 gain curves. The distribution of this bootstrap statistic was not different from normal (Kolmogorov-Smirnov test). To identify the portions of the gain curves that are significantly different from zero, we calculated a noise floor. It was calculated by cyclically shifting original spike times by a random time interval, larger than 5 correlation times, and calculating 2000 "random time-triggered averages", which were used to calculate "gain curves". The noise floor was defined as the $95^{\text {th }}$ percentile of these "gain curves". The gain curves in figures 2 and 3 were displayed either until they were crossed by the noise floor or up to $1000 \mathrm{~Hz}$, if noise floor crossing happened at a frequency $>1000 \mathrm{~Hz}$.

\section{Hilbert Analysis}

The stimulus' theta phase component was extracted by filtering with an infinite impulse response bandpass filter with 6 pole Butterworth characteristics. The filter was designed in Igor Pro 8 with pass-band limits of 3.5 and $10.5 \mathrm{~Hz}$ for the sample time of $100 \mathrm{kHz}$. Used twice, once in forward time, once in reversed time, the filter results in zero-delay filtering of the input. Fourier analysis of the input and output shows effective isolation of the 4 to $12 \mathrm{~Hz}$ component. The phase and amplitude of this component were obtained by conventional Hilbert analysis. APs were stratified according to the phase of the theta component at the AP time to perform the analysis in figure 4.

\section{Statistics}

Paired samples, two-sided Wilcoxon rank tests were performed to test the single neuron data in figures 3 and 4 . W-statistics and $p$ values are given in figures and legends. The p-values in figure 4 are not corrected for the dual comparison. 
bioRxiv preprint doi: https://doi org/10.1101/2019.12.19.882639; this version posted July 16,2021 . The copyright holder for this preprin (which was not certified by peer review) is the author/funder, who has granted bioRxiv a license to display the preprint in perpetuity. It is made available under aCC-BY-NC-ND 4.0 International license.

\section{Acknowledgments \\ Funding:}

Bundesministerium für Bildung und Forschung (BMBF, Federal Ministry of Education and Research) grant 01GQ1005B (FW, AN)

Bundesministerium für Bildung und Forschung (BMBF, Federal Ministry of Education and Research) grant 01GQ1005E (FW)

Deutsche Forschungsgemeinschaft (DFG, German Research Foundation) - 436260547, in relation to NeuroNex (NSF 2015276)

VW Foundation grant ZN2632 (FW)

GGNB Excellence Stipend of the University of Göttingen (RMM)

Max Planck Society

Author contributions:

R.M.M., C.L.P., F.W., and A.N. conceived the study. R.M.M. performed the experiments with contributions from M.M. R.M.M., C.L.P., F.W., and A.N. analyzed and interpreted the data. F.W., A.N., W.S., and J.F.S. provided resources. All authors discussed and interpreted the data. R.M.M., F.W, and A.N. wrote the paper with inputs from all other authors.

Competing interests: Authors declare no competing interest.

Data and materials availability: All data are available from the corresponding author upon reasonable request. Raw data underlying the dynamic gain curves can be downloaded from this permanent repository at the Max Planck Digital Library: https://edmond.mpdl.mpg.de/imeji/collection/pdxNFpqJurbDDeop.

This permalink is for review purposes. It will be replaced with a DOI.

The code, written in IgorPro 8.0 and 9.0, used to analyze raw data and generate the dynamic gain curves, is included in the data repository. The code is continuously maintained. The latest version is available at https://github.com/Anneef/AnTools.

References

1. G. Buzsaki, A. Draguhn, Neuronal oscillations in cortical networks. Science 304, 1926-1929 (2004); published online EpubJun 25 (10.1126/science.1099745).

2. J. Cannon, M. M. McCarthy, S. Lee, J. Lee, C. Borgers, M. A. Whittington, N. Kopell, Neurosystems: brain rhythms and cognitive processing. Eur J Neurosci 39, 705-719 (2014); published online EpubMar (10.1111/ejn.12453).

3. J. E. Lisman, O. Jensen, The theta-gamma neural code. Neuron 77, 1002-1016 (2013); published online EpubMar 20 (10.1016/j.neuron.2013.03.007).

4. J. A. Cardin, M. Carlen, K. Meletis, U. Knoblich, F. Zhang, K. Deisseroth, L. H. Tsai, C. I. Moore, Driving fast-spiking cells induces gamma rhythm and controls sensory responses. Nature 459, 663-667 (2009); published online EpubJun 4 (10.1038/nature08002).

5. M. A. Whittington, R. D. Traub, J. G. Jefferys, Synchronized oscillations in interneuron networks driven by metabotropic glutamate receptor activation. Nature 373, 612-615 (1995); published online EpubFeb 16 (10.1038/373612a0).

6. F. G. Pike, R. S. Goddard, J. M. Suckling, P. Ganter, N. Kasthuri, O. Paulsen, Distinct frequency preferences of different types of rat hippocampal neurones in response to oscillatory input currents. J Physiol 529 Pt 1, 205-213 (2000); published online EpubNov 15 (

7. J. Veit, R. Hakim, M. P. Jadi, T. J. Sejnowski, H. Adesnik, Cortical gamma band synchronization through somatostatin interneurons. Nat Neurosci 20, 951-959 (2017); published online EpubJul (10.1038/nn.4562). 
bioRxiv preprint doi: https://doi.org/10.1101/2019.12.19.882639; this version posted July 16 , 2021. The copyright holder for this preprint (which was not certified by peer review) is the author/funder, who has granted bioRxiv a license to display the preprint in perpetuity. It is made available under aCC-BY-NC-ND 4.0 International license.

8. R. Hakim, K. Shamardani, H. Adesnik, A neural circuit for gamma-band coherence across the retinotopic map in mouse visual cortex. Elife 7, (2018); published online EpubFeb 26 (10.7554/eLife.28569).

9. G. Chen, Y. Zhang, X. Li, X. Zhao, Q. Ye, Y. Lin, H. W. Tao, M. J. Rasch, X. Zhang, Distinct Inhibitory Circuits Orchestrate Cortical beta and gamma Band Oscillations. Neuron 96, 1403-1418 e1406 (2017); published online EpubDec 20 (10.1016/j.neuron.2017.11.033).

10. H. Kondgen, C. Geisler, S. Fusi, X. J. Wang, H. R. Luscher, M. Giugliano, The dynamical response properties of neocortical neurons to temporally modulated noisy inputs in vitro. Cereb Cortex 18, 2086-2097 (2008); published online EpubSep (10.1093/cercor/bhm235).

11. E. Lazarov, M. Dannemeyer, B. Feulner, J. Enderlein, M. J. Gutnick, F. Wolf, A. Neef, An axon initial segment is required for temporal precision in action potential encoding by neuronal populations. Science advances 4, eaau8621 (2018); published online EpubNov (10.1126/sciadv.aau8621).

12. M. H. Higgs, W. J. Spain, Conditional bursting enhances resonant firing in neocortical layer 2-3 pyramidal neurons. J Neurosci 29, 1285-1299 (2009); published online EpubFeb 4 (10.1523/JNEUROSCI.3728-08.2009).

13. N. Brunel, F. S. Chance, N. Fourcaud, L. F. Abbott, Effects of synaptic noise and filtering on the frequency response of spiking neurons. Phys Rev Lett 86, 2186-2189 (2001); published online EpubMar 5 (

14. B. Naundorf, F. Wolf, M. Volgushev, Unique features of action potential initiation in cortical neurons. Nature 440, 1060-1063 (2006); published online EpubApr 20 (10.1038/nature04610).

15. J. F. A. Poulet, S. Crochet, The Cortical States of Wakefulness. Frontiers in systems neuroscience 12, 64 (2018)10.3389/fnsys.2018.00064).

16. D. Feldmeyer, G. Qi, V. Emmenegger, J. F. Staiger, Inhibitory interneurons and their circuit motifs in the many layers of the barrel cortex. Neuroscience 368, 132-151 (2018); published online EpubJan 1 (10.1016/j.neuroscience.2017.05.027).

17. A. Destexhe, M. Rudolph, D. Pare, The high-conductance state of neocortical neurons in vivo. Nat Rev Neurosci 4, 739-751 (2003); published online EpubSep (10.1038/nrn1198).

18. R. T. Canolty, R. T. Knight, The functional role of cross-frequency coupling. Trends Cogn Sci 14, 506-515 (2010); published online EpubNov (10.1016/j.tics.2010.09.001).

19. N. Brunel, V. Hakim, Fast global oscillations in networks of integrate-and-fire neurons with low firing rates. Neural Comput 11, 1621-1671 (1999); published online EpubOct $1(10.1162 / 089976699300016179)$.

20. C. Geisler, N. Brunel, X. J. Wang, Contributions of intrinsic membrane dynamics to fast network oscillations with irregular neuronal discharges. J Neurophysiol 94, 43444361 (2005); published online EpubDec (10.1152/jn.00510.2004).

21. T. Tchumatchenko, A. Malyshev, F. Wolf, M. Volgushev, Ultrafast population encoding by cortical neurons. J Neurosci 31, 12171-12179 (2011); published online EpubAug 24 (10.1523/JNEUROSCI.2182-11.2011).

22. H. R. Joo, L. M. Frank, The hippocampal sharp wave-ripple in memory retrieval for immediate use and consolidation. Nat Rev Neurosci 19, 744-757 (2018); published online EpubDec (10.1038/s41583-018-0077-1).

23. A. H. Meyer, I. Katona, M. Blatow, A. Rozov, H. Monyer, In vivo labeling of parvalbumin-positive interneurons and analysis of electrical coupling in identified neurons. J Neurosci 22, 7055-7064 (2002); published online EpubAug 15 (20026742). 